\title{
Genetically determined high activities of the TNF-alpha, IL23/IL17, and NFkB pathways were associated with increased risk of ankylosing spondylitis
}

Jacob Sode ${ }^{1,2,3,4}$, Steffen Bank ${ }^{5,6^{*}}$ (D), Ulla Vogel ${ }^{7}$, Paal Skytt Andersen ${ }^{8,9}$, Signe Bek Sørensen ${ }^{1,5,10}$, Anders Bo Bojesen ${ }^{5}$, Malene Rohr Andersen ${ }^{11}$, Ivan Brandslund ${ }^{12}$, Ram Benny Dessau ${ }^{13}$, Hans Jürgen Hoffmann ${ }^{14,15}$, Bente Glintborg ${ }^{16,17}$, Merete Lund Hetland ${ }^{17,18}$, Henning Locht ${ }^{3}$, Niels Henrik Heegaard ${ }^{2,19^{\wedge}}$ and Vibeke Andersen ${ }^{1,5,10,20}$

\section{Abstract}

Background: Ankylosing spondylitis (AS) results from the combined effects of susceptibility genes and environmental factors. Polymorphisms in genes regulating inflammation may explain part of the heritability of AS.

Methods: Using a candidate gene approach in this case-control study, 51 mainly functional single nucleotide polymorphisms (SNPs) in genes regulating inflammation were assessed in 709 patients with AS and 795 controls. Data on the patients with AS were obtained from the DANBIO registry where patients from all of Denmark are monitored in routine care during treatment with conventional and biologic disease modifying anti-rheumatic drugs (bDMARDs). The results were analyzed using logistic regression (adjusted for age and sex).

Results: Nine polymorphisms were associated with risk of AS $(p<0.05)$. The polymorphisms were in genes regulating a: the TNF-a pathway (TNF-308 G > A (rs1800629), and - 238 G > A (rs361525); TNFRSF1A -609 G > T (rs4149570), and PTPN22 $1858 \mathrm{G}>\mathrm{A}(\mathrm{rs} 2476601)$ ), b: the IL23/IL17 pathway (IL23R G > A (rs11209026), and IL18-137 G >C (rs187238)), or c: the NFkB pathway (TLR1 743 T > C (rs4833095), TLR4 T > C (rs1554973), and LY96-1625 C > G (rs1 1465996)). After Bonferroni correction the homozygous variant genotype of TLR1 743 T > C (rs4833095) (odds ratios (OR): 2.59, 95\% confidence interval (Cl): 1.48-4.51, $p=0.04$ ), and TNFRSF1A -609 G > T (rs4149570) (OR: 1.79, 95\% Cl: 1.31-2.41, $p=0.01$ ) were associated with increased risk of AS and the combined homozygous and heterozygous variant genotypes of TNF -308 G > A (rs1800629) (OR: 0.56, 95\% Cl: 0.44-0.72, $p=0.0002$ ) were associated with reduced risk of AS.

Conclusion: We replicated associations between AS and the polymorphisms in TNF (rs1800629), TNFRSF1A (rs4149570), and IL23R (rs11209026). Furthermore, we identified novel risk loci in TNF (rs361525), IL 18 (rs187238), TLR1 (rs4833095), TLR4 (rs1554973), and LY96 (rs11465996) that need validation in independent cohorts. The results suggest that genetically determined high activity of the TNF-a, IL23/LL17, and NFkB pathways increase risk of AS.

Keywords: Ankylosing spondylitis, Single nucleotide polymorphism, SNP, Case-control study

\footnotetext{
*Correspondence: stb@mb.au.dk

Deceased

${ }^{5}$ Focused Research Unit for Molecular Diagnostic and Clinical Research,

Hospital of Southern Jutland, Aabenraa, Denmark

${ }^{6}$ Medical Department, Viborg Regional Hospital, Viborg, Denmark

Full list of author information is available at the end of the article
}

(c) The Author(s). 2018 Open Access This article is distributed under the terms of the Creative Commons Attribution 4.0 International License (http://creativecommons.org/licenses/by/4.0/), which permits unrestricted use, distribution, and reproduction in any medium, provided you give appropriate credit to the original author(s) and the source, provide a link to the Creative Commons license, and indicate if changes were made. The Creative Commons Public Domain Dedication waiver (http://creativecommons.org/publicdomain/zero/1.0/) applies to the data made available in this article, unless otherwise stated. 


\section{Background}

Ankylosing spondylitis (AS) is a type of spondyloarthritis in which hallmark clinical features are inflammation at entheses and subchondral bone of the pelvic and spinal joints with subsequent abnormal new bone formation at these sites. Ultimately, this leads to ossification of entheses and joints resulting in loss of joint mobility. The incidence varies between 0.1 and $1.8 \%$ with the highest incidence in Scandinavia. Onset is typically in young adults with a male predominance. Medications used include non-steroid anti-inflammatory drugs (NSAIDs), and biological diseasemodifying anti-rheumatic drugs (bDMARDs), i.e. tumor necrosis factor- $\alpha$ inhibitors (anti-TNF) and more recently also an interleukin(IL)-17A inhibitor (secukinumab) [1].

The cause of AS is unknown but is believed to involve a combination of genetic and environmental factors [2]. The heritability is polygenic and estimated to exceed 90\%, with the HLA-B27 allele as the major contributor accounting for approximately $25 \%$ of the heritability of AS [2]. The IL-17/ IL-23 pathway and the TNF- $\alpha$ pathway are central in the pathogenesis of AS and alterations in these pathways have been shown in mouse models to affect development and severity of enthesitis $[3,4]$.

TNF- $\alpha$ can be activated by Pathogen-Associated Molecular Patterns (PAMPs) such as bacterial or viral DNA, flagellin, or lipopolysaccharide (LPS), through the NFkB pathway. PAMPs can be recognized by Toll-like receptors (TLRs) thereby initiating a kinase cascade which phosphorylates and degrades the NFkB inhibitor IkB $\alpha$ [5]. This releases NFkB which is transported from the cytosol to the nucleus where it initiates expression of pro- and anti-inflammatory cytokines including TNF- $\alpha$ and IL-17 (http://www.bu.edu/nf-kb/gene-resources/targetgenes/). The TNF- $\alpha$ and NFkB pathway are intertwined and TNF- $\alpha$ can feedback stimulate NFkB by binding to TNF receptors (TNFR1 or TNFR2), resulting in a kinase cascade similar to, but distinct from, the pathway induced by TLRs [5].

The IL23/IL17 pathway can also stimulate TNF- $\alpha$ activity. The pro-inflammatory cytokine IL-17 enhances the production of other pro-inflammatory cytokines including TNF- $\alpha$, and the secretion IL-17 itself can be enhanced by IL-23 [6].

PAMPs can also be recognized by intracellular Nodlike receptors (NLRs). In turn, NLRs can activate proinflammatory cytokines including IL-18 [7]. IL-18 is invloved in the IL23/IL17 pathway and can enhance the production of IL-17 [8].

The aim of this study was to assess whether functional single nucleotide polymorphisms.

(SNPs) in genes involved in the TNF- $\alpha$, IL23/IL17, $\mathrm{NFkB}$, and other pro- and anti-inflammatory pathways were associated with risk of AS.

\section{Methods}

\section{Patients and samples}

The DANBIO registry includes prospectively collected clinical data on patients with inflammatory joint diseases including smoking status, disease characteristics e.g. HLA-B27 status, disease activity, treatment, and treatment outcomes. Patients from all of Denmark are monitored in routine care during treatment with conventional and biologic disease modifying anti-rheumatic drugs (bDMARDs) [9].

Screening for tuberculosis before initiation of treatment with biological drugs is routinely performed in Denmark. Left over blood clots (after whole blood analysis for Mycobacterium tuberculosis) were collected from all patients screened for tuberculosis at Statens Serum Institut (Copenhagen, Denmark) from 01.09.2009 to 31.01.2013; the Department of Respiratory Diseases B and the Department of Clinical Microbiology, Aarhus University Hospital (Aarhus, Denmark) from 01.01.2011 to 31.01.2014; the Department of Clinical Biochemistry, Herlev and Gentofte Hospital (Hellerup, Denmark) from 01.03.2012 to 31.01.2014; the Department of Biochemistry, Hospital of Lillebaelt (Vejle, Denmark); and the Department of Biochemistry, Hospital of Slagelse (Slagelse, Denmark) from 01.01.2014 to 31.01.2014. Furthermore, from 01.01.2013 to 31.12.2013 blood samples were collected from all patients with AS treated with or without anti-TNF drugs at the Department of Rheumatology, Frederiksberg Hospital (Frederiksberg, Denmark).

By linking the unique personal identification number of Danish citizens (CPR-number) from each blood sample with the clinical data from DANBIO, 709 patients with AS (ICD-10: M45.9) were identified. The control group consisted of 795 healthy blood donors recruited from Viborg, Denmark.

\section{Genotyping}

Fifty-one SNPs in genes involved in the TNF- $\alpha$, IL23/ IL17, NFKB, and other pro- and anti-inflammatory pathways were assessed. A list of all SNPs studied and genotype distribution is presented in Table 1 and SNPs associated with AS are summarized in Table 2.

DNA extraction (Maxwell 16 LEV Blood DNA Kit; Promega, Madison, WI, USA) was performed as described by Bank et al. [10]. For the healthy controls, DNA was extracted from EDTA-stabilized peripheral blood by either PureGene (Qiagen, Hilden, Germany) or Wizard Genomic (Promega, Madison, Wisconsin, USA) DNA purification kit according to the manufacturers` instructions [11-17]. Competitive Allele-Specific Polymerase chain reaction $\left(\mathrm{KASP}^{\mathrm{TM}}\right)$, an end-point PCR technology, was used by LGC Genomics for genotyping (LGC Genomics, Hoddesdon, United Kingdom) (http:// www.lgcgenomics.com/). 
Table 1 Odds ratios (OR) and 95\% confidence interval (95Cl) for genotypes studied among healthy controls and patients with ankylosing spondylitis (AS)

\begin{tabular}{|c|c|c|c|c|c|c|c|c|}
\hline \multirow{2}{*}{$\begin{array}{l}\text { Gene } \\
\text { rs-number }\end{array}$} & \multirow[t]{2}{*}{ Healthy controls } & \multirow[t]{2}{*}{ AS } & \multicolumn{2}{|l|}{ Unadjusted } & \multicolumn{2}{|c|}{ Adjusted, age \& sex } & \multicolumn{2}{|c|}{ Adjusted, age, sex \& smoking } \\
\hline & & & $\mathrm{OR}(95 \mathrm{Cl})$ & p & OR (9 5Cl) & $p$ & OR $(95 \mathrm{Cl})$ & $p$ \\
\hline \multicolumn{9}{|l|}{$\begin{array}{l}\text { TLR1 } \\
\text { rs4833095 }\end{array}$} \\
\hline$\pi$ & 485 & 415 & & & & & & \\
\hline $\mathrm{TC}$ & 261 & 238 & $1.07(0.86-1.33)$ & 0.57 & $1.03(0.82-1.29)$ & 0.83 & $1.05(0.78-1.42)$ & 0.73 \\
\hline $\mathrm{CC}$ & 20 & 43 & $2.51(1.45-4.34)$ & 0.00095 & $2.59(1.48-4.51)$ & 0.00081 & $2.86(1.44-5.68)$ & 0.0026 \\
\hline TC or CC & 281 & 281 & $1.17(0.95-1.44)$ & 0.15 & $1.14(0.91-1.41)$ & 0.25 & $1.18(0.89-1.58)$ & 0.26 \\
\hline \multicolumn{9}{|l|}{$\begin{array}{l}\text { TLR2 } \\
\text { rs3804099 }\end{array}$} \\
\hline$\pi$ & 241 & 197 & & & & & & \\
\hline $\mathrm{TC}$ & 393 & 354 & $1.10(0.87-1.40)$ & 0.42 & $1.07(0.84-1.37)$ & 0.58 & $1.02(0.73-1.42)$ & 0.90 \\
\hline CC & 144 & 142 & $1.21(0.89-1.63)$ & 0.22 & $1.24(0.91-1.68)$ & 0.17 & $1.30(0.87-1.96)$ & 0.20 \\
\hline TC or CC & 537 & 496 & $1.13(0.90-1.41)$ & 0.29 & $1.11(0.89-1.40)$ & 0.36 & $1.10(0.80-1.50)$ & 0.57 \\
\hline \multicolumn{9}{|l|}{$\begin{array}{l}\text { TLR2 } \\
\text { rs 11938228 }\end{array}$} \\
\hline CC & 327 & 314 & & & & & & \\
\hline CA & 368 & 313 & $0.89(0.71-1.10)$ & 0.27 & $0.86(0.69-1.07)$ & 0.17 & $0.80(0.60-1.08)$ & 0.15 \\
\hline $\mathrm{AA}$ & 76 & 69 & $0.95(0.66-1.36)$ & 0.76 & $0.92(0.63-1.33)$ & 0.66 & $1.03(0.62-1.69)$ & 0.92 \\
\hline CA or $A A$ & 444 & 382 & $0.90(0.73-1.10)$ & 0.30 & $0.87(0.70-1.07)$ & 0.19 & $0.84(0.63-1.11)$ & 0.22 \\
\hline \multicolumn{9}{|l|}{$\begin{array}{l}\text { TLR2 } \\
\text { rs4696480 }\end{array}$} \\
\hline $\mathrm{AA}$ & 199 & 179 & & & & & & \\
\hline AT & 417 & 348 & $0.93(0.72-1.19)$ & 0.55 & $0.89(0.69-1.15)$ & 0.38 & $0.84(0.60-1.18)$ & 0.31 \\
\hline$\pi$ & 155 & 169 & $1.21(0.90-1.63)$ & 0.20 & $1.16(0.86-1.58)$ & 0.33 & $1.18(0.78-1.78)$ & 0.44 \\
\hline AT or TT & 572 & 517 & $1.00(0.79-1.27)$ & 0.97 & $0.97(0.76-1.23)$ & 0.78 & $0.92(0.67-1.27)$ & 0.62 \\
\hline \multicolumn{9}{|l|}{$\begin{array}{l}\text { TLR4 } \\
\text { rs5030728 }\end{array}$} \\
\hline GG & 359 & 322 & & & & & & \\
\hline GA & 323 & 298 & $1.03(0.83-1.28)$ & 0.80 & $1.01(0.81-1.27)$ & 0.91 & $0.93(0.69-1.25)$ & 0.62 \\
\hline $\mathrm{AA}$ & 78 & 70 & $1.00(0.70-1.43)$ & 1.00 & $0.98(0.68-1.42)$ & 0.93 & $0.87(0.53-1.42)$ & 0.57 \\
\hline GA or $A A$ & 401 & 368 & $1.02(0.83-1.26)$ & 0.83 & $1.01(0.82-1.25)$ & 0.94 & $0.91(0.69-1.21)$ & 0.53 \\
\hline \multicolumn{9}{|l|}{$\begin{array}{l}\text { TLR4 } \\
\text { rs1554973 }\end{array}$} \\
\hline$\pi$ & 440 & 395 & & & & & & \\
\hline $\mathrm{TC}$ & 272 & 261 & $1.07(0.86-1.33)$ & 0.55 & $1.06(0.85-1.32)$ & 0.62 & $0.98(0.73-1.32)$ & 0.90 \\
\hline CC & 62 & 33 & $0.59(0.38-0.92)$ & 0.02 & $0.55(0.34-0.86)$ & 0.01 & $0.68(0.38-1.23)$ & 0.20 \\
\hline TC or CC & 334 & 294 & $0.98(0.80-1.21)$ & 0.85 & $0.96(0.78-1.19)$ & 0.72 & $0.93(0.70-1.24)$ & 0.63 \\
\hline \multicolumn{9}{|l|}{$\begin{array}{l}\text { TLR4 } \\
\text { rs12377632 }\end{array}$} \\
\hline$\pi$ & 306 & 271 & & & & & & \\
\hline $\mathrm{TC}$ & 358 & 319 & $1.01(0.81-1.26)$ & 0.96 & $1.05(0.84-1.32)$ & 0.66 & $1.07(0.78-1.46)$ & 0.67 \\
\hline $\mathrm{CC}$ & 102 & 96 & $1.06(0.77-1.47)$ & 0.71 & $1.11(0.80-1.55)$ & 0.52 & $1.41(0.92-2.17)$ & 0.12 \\
\hline TC or CC & 460 & 415 & $1.02(0.83-1.26)$ & 0.86 & $1.06(0.86-1.32)$ & 0.58 & $1.14(0.85-1.53)$ & 0.37 \\
\hline \multicolumn{9}{|l|}{$\begin{array}{l}\text { TLR5 } \\
\text { rs5744168 }\end{array}$} \\
\hline $\mathrm{CC}$ & 672 & 605 & & & & & & \\
\hline
\end{tabular}


Table 1 Odds ratios (OR) and 95\% confidence interval (95Cl) for genotypes studied among healthy controls and patients with ankylosing spondylitis (AS) (Continued)

\begin{tabular}{|c|c|c|c|c|c|c|c|c|}
\hline \multirow{2}{*}{$\begin{array}{l}\text { Gene } \\
\text { rs-number }\end{array}$} & \multirow[t]{2}{*}{ Healthy controls } & \multirow[t]{2}{*}{ AS } & \multicolumn{2}{|l|}{ Unadjusted } & \multicolumn{2}{|c|}{ Adjusted, age \& sex } & \multicolumn{2}{|c|}{ Adjusted, age, sex \& smoking } \\
\hline & & & $\mathrm{OR}(95 \mathrm{Cl})$ & $p$ & OR $(95 \mathrm{Cl})$ & $p$ & OR $(95 \mathrm{Cl})$ & $p$ \\
\hline CT & 94 & 89 & $1.05(0.77-1.43)$ & 0.75 & $1.05(0.77-1.45)$ & 0.74 & $0.89(0.58-1.37)$ & 0.60 \\
\hline$\pi$ & 5 & 2 & $0.44(0.09-2.30)$ & 0.33 & $0.45(0.08-2.43)$ & 0.35 & $0.04(0.00-3.54)$ & 0.16 \\
\hline $\mathrm{CT}$ or $\mathrm{TT}$ & 99 & 91 & $1.02(0.75-1.39)$ & 0.89 & $1.02(0.75-1.40)$ & 0.88 & $0.84(0.55-1.29)$ & 0.43 \\
\hline \multicolumn{9}{|l|}{$\begin{array}{l}\text { TLR5 } \\
\text { rs5744174 }\end{array}$} \\
\hline$\pi$ & 215 & 216 & & & & & & \\
\hline TC & 399 & 337 & $0.84(0.66-1.07)$ & 0.15 & $0.85(0.67-1.09)$ & 0.20 & $0.82(0.60-1.14)$ & 0.24 \\
\hline CC & 144 & 138 & $0.95(0.71-1.29)$ & 0.76 & $1.02(0.75-1.39)$ & 0.91 & $0.87(0.57-1.32)$ & 0.51 \\
\hline TC or CC & 543 & 475 & $0.87(0.69-1.09)$ & 0.23 & $0.90(0.71-1.13)$ & 0.36 & $0.84(0.62-1.14)$ & 0.26 \\
\hline \multicolumn{9}{|l|}{$\begin{array}{l}\text { TLR9 } \\
\text { rs } 187084\end{array}$} \\
\hline$\pi$ & 262 & 237 & & & & & & \\
\hline $\mathrm{TC}$ & 366 & 335 & $1.01(0.80-1.27)$ & 0.92 & $1.03(0.82-1.31)$ & 0.78 & $1.09(0.79-1.50)$ & 0.60 \\
\hline CC & 142 & 120 & $0.93(0.69-1.26)$ & 0.66 & $0.91(0.67-1.24)$ & 0.56 & $1.07(0.71-1.61)$ & 0.76 \\
\hline TC or CC & 508 & 455 & $0.99(0.80-1.23)$ & 0.93 & $1.00(0.80-1.25)$ & 0.98 & $1.08(0.80-1.46)$ & 0.60 \\
\hline \multicolumn{9}{|l|}{$\begin{array}{l}\text { TLR9 } \\
\text { rs352139 }\end{array}$} \\
\hline GG & 255 & 211 & & & & & & \\
\hline GA & 347 & 324 & $1.13(0.89-1.43)$ & 0.32 & $1.08(0.85-1.38)$ & 0.52 & $1.01(0.73-1.40)$ & 0.93 \\
\hline AA & 167 & 139 & $1.01(0.75-1.34)$ & 0.97 & $0.96(0.71-1.30)$ & 0.79 & $0.80(0.53-1.20)$ & 0.27 \\
\hline GA or $A A$ & 514 & 463 & $1.09(0.87-1.36)$ & 0.45 & $1.04(0.83-1.31)$ & 0.72 & $0.94(0.69-1.27)$ & 0.68 \\
\hline \multicolumn{9}{|l|}{$\begin{array}{l}\text { LY96 } \\
\text { rs11465996 }\end{array}$} \\
\hline $\mathrm{CC}$ & 344 & 341 & & & & & & \\
\hline CG & 337 & 298 & $0.89(0.72-1.11)$ & 0.30 & $0.91(0.73-1.14)$ & 0.42 & $0.89(0.66-1.20)$ & 0.45 \\
\hline GG & 81 & 53 & $0.66(0.45-0.96)$ & 0.03 & $0.68(0.46-1.00)$ & 0.0498 & $0.65(0.39-1.10)$ & 0.11 \\
\hline CG or GG & 418 & 351 & $0.85(0.69-1.04)$ & 0.11 & $0.87(0.70-1.07)$ & 0.18 & $0.84(0.63-1.12)$ & 0.24 \\
\hline \multicolumn{9}{|l|}{$\begin{array}{l}\text { CD14 } \\
\text { Rs2569190 }\end{array}$} \\
\hline GG & 236 & 194 & & & & & & \\
\hline GA & 360 & 339 & $1.15(0.90-1.46)$ & 0.27 & $1.18(0.92-1.51)$ & 0.19 & $1.27(0.91-1.78)$ & 0.16 \\
\hline $\mathrm{AA}$ & 170 & 157 & $1.12(0.84-1.50)$ & 0.43 & $1.20(0.89-1.61)$ & 0.24 & $1.46(0.98-2.19)$ & 0.06 \\
\hline GA or $A A$ & 530 & 496 & $1.14(0.91-1.43)$ & 0.26 & $1.18(0.94-1.50)$ & 0.15 & $1.32(0.96-1.82)$ & 0.08 \\
\hline \multicolumn{9}{|l|}{$\begin{array}{l}\text { TIRAP } \\
\text { rs8177374 }\end{array}$} \\
\hline$C C$ & 556 & 521 & & & & & & \\
\hline CT & 185 & 159 & $0.92(0.72-1.17)$ & 0.49 & $0.99(0.77-1.27)$ & 0.94 & $1.38(0.99-1.91)$ & 0.06 \\
\hline$\pi$ & 21 & 15 & $0.76(0.39-1.49)$ & 0.43 & $0.76(0.38-1.53)$ & 0.45 & $1.31(0.55-3.12)$ & 0.55 \\
\hline $\mathrm{CT}$ or $\mathrm{TT}$ & 206 & 174 & $0.90(0.71-1.14)$ & 0.39 & $0.97(0.76-1.23)$ & 0.81 & $1.38(1.00-1.89)$ & 0.047 \\
\hline \multicolumn{9}{|l|}{$\begin{array}{l}\text { SUMO4 } \\
\text { rs } 237025\end{array}$} \\
\hline$\pi$ & 215 & 195 & & & & & & \\
\hline $\mathrm{TC}$ & 362 & 358 & $1.09(0.86-1.39)$ & 0.48 & $1.08(0.84-1.38)$ & 0.55 & $1.04(0.75-1.44)$ & 0.80 \\
\hline $\mathrm{CC}$ & 195 & 136 & $0.77(0.57-1.03)$ & 0.08 & $0.75(0.55-1.01)$ & 0.06 & $0.55(0.36-0.84)$ & 0.01 \\
\hline TC or CC & 557 & 494 & $0.98(0.78-1.23)$ & 0.85 & $0.96(0.76-1.22)$ & 0.75 & $0.87(0.64-1.19)$ & 0.38 \\
\hline
\end{tabular}


Table 1 Odds ratios (OR) and 95\% confidence interval (95Cl) for genotypes studied among healthy controls and patients with ankylosing spondylitis (AS) (Continued)

\begin{tabular}{|c|c|c|c|c|c|c|c|c|}
\hline \multirow{2}{*}{$\begin{array}{l}\text { Gene } \\
\text { rs-number }\end{array}$} & \multirow[t]{2}{*}{ Healthy controls } & \multirow[t]{2}{*}{ AS } & \multicolumn{2}{|l|}{ Unadjusted } & \multicolumn{2}{|c|}{ Adjusted, age \& sex } & \multicolumn{2}{|c|}{ Adjusted, age, sex \& smoking } \\
\hline & & & $\mathrm{OR}(95 \mathrm{Cl})$ & p & OR $(95 \mathrm{Cl})$ & $p$ & OR $(95 \mathrm{Cl})$ & $p$ \\
\hline \multicolumn{9}{|l|}{$\begin{array}{l}\text { NFKBIA } \\
\text { rs696 }\end{array}$} \\
\hline GG & 298 & 259 & & & & & & \\
\hline GA & 366 & 336 & $1.06(0.85-1.32)$ & 0.63 & $1.06(0.84-1.33)$ & 0.64 & $1.02(0.75-1.39)$ & 0.88 \\
\hline AA & 101 & 90 & $1.03(0.74-1.43)$ & 0.88 & $0.97(0.69-1.36)$ & 0.86 & $1.07(0.67-1.69)$ & 0.78 \\
\hline GA or $A A$ & 467 & 426 & $1.05(0.85-1.30)$ & 0.65 & $1.04(0.84-1.29)$ & 0.73 & $1.03(0.77-1.38)$ & 0.84 \\
\hline \multicolumn{9}{|l|}{$\begin{array}{l}\text { NFKB1 } \\
\text { rs28362491 }\end{array}$} \\
\hline Ins/Ins & 269 & 258 & & & & & & \\
\hline Ins/- & 376 & 316 & $0.88(0.70-1.10)$ & 0.25 & $0.89(0.70-1.12)$ & 0.31 & $0.74(0.54-1.01)$ & 0.06 \\
\hline$-/-$ & 122 & 100 & $0.85(0.62-1.17)$ & 0.33 & $0.82(0.59-1.13)$ & 0.22 & $0.78(0.51-1.19)$ & 0.25 \\
\hline Ins/- or -/- & 498 & 416 & $0.87(0.70-1.08)$ & 0.21 & $0.87(0.70-1.08)$ & 0.21 & $0.75(0.56-1.01)$ & 0.06 \\
\hline \multicolumn{9}{|l|}{$\begin{array}{l}\text { TNF } \\
\text { rs1800629 }\end{array}$} \\
\hline GG & 527 & 549 & & & & & & \\
\hline GA & 223 & 129 & $0.56(0.43-0.71)$ & 0.0000032 & $0.58(0.45-0.75)$ & 0.000029 & $0.63(0.45-0.89)$ & 0.01 \\
\hline $\mathrm{AA}$ & 25 & 9 & $0.35(0.16-0.75)$ & 0.01 & $0.39(0.18-0.85)$ & 0.02 & $0.19(0.04-0.79)$ & 0.02 \\
\hline GA or $\mathrm{AA}$ & 248 & 138 & $0.53(0.42-0.68)$ & 0.00000030 & $0.56(0.44-0.72)$ & 0.0000047 & $0.59(0.42-0.82)$ & 0.0018 \\
\hline \multicolumn{9}{|l|}{$\begin{array}{l}\text { TNF } \\
\text { rs361525 }\end{array}$} \\
\hline GG & 708 & 669 & & & & & & \\
\hline GA & 60 & 30 & $0.53(0.34-0.83)$ & 0.01 & $0.52(0.32-0.82)$ & 0.0049 & $0.61(0.33-1.12)$ & 0.11 \\
\hline $\mathrm{AA}$ & 3 & 0 & $1.00(1.00-1.00)$ & 1.00 & $1.00(1.00-1.00)$ & 1.00 & $1.00(1.00-1.00)$ & 1.00 \\
\hline GA or $\mathrm{AA}$ & 63 & 30 & $0.50(0.32-0.79)$ & 0.0027 & $0.49(0.31-0.78)$ & 0.0024 & $0.58(0.32-1.05)$ & 0.07 \\
\hline \multicolumn{9}{|l|}{$\begin{array}{l}\text { TNFRSF1A } \\
\text { rs4149570 }\end{array}$} \\
\hline GG & 307 & 217 & & & & & & \\
\hline GT & 355 & 339 & $1.35(1.07-1.70)$ & 0.01 & $1.33(1.05-1.68)$ & 0.02 & $1.46(1.06-2.00)$ & 0.02 \\
\hline$\pi$ & 109 & 132 & $1.71(1.26-2.33)$ & 0.00060 & $1.79(1.31-2.46)$ & 0.00027 & $2.26(1.48-3.47)$ & 0.00017 \\
\hline GT or TT & 464 & 471 & $1.44(1.16-1.78)$ & 0.0010 & $1.44(1.15-1.80)$ & 0.0013 & $1.64(1.21-2.22)$ & 0.0014 \\
\hline \multicolumn{9}{|l|}{$\begin{array}{l}\text { TNFAIP3 } \\
\text { rs6927172 }\end{array}$} \\
\hline $\mathrm{CC}$ & 473 & 415 & & & & & & \\
\hline$C G$ & 264 & 245 & $1.06(0.85-1.32)$ & 0.61 & $1.06(0.85-1.33)$ & 0.61 & $1.03(0.76-1.39)$ & 0.85 \\
\hline GG & 40 & 25 & $0.71(0.42-1.19)$ & 0.20 & $0.70(0.41-1.19)$ & 0.18 & $0.51(0.23-1.10)$ & 0.09 \\
\hline CG or GG & 304 & 270 & $1.01(0.82-1.25)$ & 0.91 & $1.01(0.82-1.26)$ & 0.91 & $0.95(0.71-1.27)$ & 0.73 \\
\hline \multicolumn{9}{|l|}{$\begin{array}{l}\text { TGFB1 } \\
\text { rs1800469 }\end{array}$} \\
\hline $\mathrm{CC}$ & 383 & 344 & & & & & & \\
\hline$C T$ & 297 & 299 & $1.12(0.90-1.39)$ & 0.30 & $1.08(0.87-1.35)$ & 0.48 & $1.28(0.95-1.71)$ & 0.11 \\
\hline$\Pi$ & 86 & 53 & $0.69(0.47-1.00)$ & 0.047 & $0.69(0.47-1.02)$ & 0.06 & $0.69(0.40-1.17)$ & 0.17 \\
\hline $\mathrm{CT}$ or $\mathrm{TT}$ & 383 & 352 & $1.02(0.83-1.26)$ & 0.83 & $1.00(0.81-1.23)$ & 0.97 & $1.14(0.86-1.52)$ & 0.35 \\
\hline \multicolumn{9}{|l|}{$\begin{array}{l}\text { PTPN22 } \\
\text { rs2476601 }\end{array}$} \\
\hline GG & 588 & 557 & & & & & & \\
\hline
\end{tabular}


Table 1 Odds ratios (OR) and 95\% confidence interval (95Cl) for genotypes studied among healthy controls and patients with ankylosing spondylitis (AS) (Continued)

\begin{tabular}{|c|c|c|c|c|c|c|c|c|}
\hline \multirow{2}{*}{$\begin{array}{l}\text { Gene } \\
\text { rs-number }\end{array}$} & \multirow[t]{2}{*}{ Healthy controls } & \multirow[t]{2}{*}{ AS } & \multicolumn{2}{|l|}{ Unadjusted } & \multicolumn{2}{|c|}{ Adjusted, age \& sex } & \multicolumn{2}{|c|}{ Adjusted, age, sex \& smoking } \\
\hline & & & OR (95 Cl) & $p$ & $\overline{O R}(95 \mathrm{Cl})$ & $p$ & $\overline{\mathrm{OR}}(95 \mathrm{Cl})$ & $p$ \\
\hline GA & 166 & 122 & $0.78(0.60-1.01)$ & 0.06 & $0.77(0.59-1.00)$ & 0.05 & $0.75(0.52-1.09)$ & 0.13 \\
\hline AA & 11 & 6 & $0.58(0.21-1.57)$ & 0.28 & $0.57(0.20-1.58)$ & 0.28 & $0.83(0.21-3.28)$ & 0.80 \\
\hline GA or $A A$ & 177 & 128 & $0.76(0.59-0.99)$ & 0.04 & $0.76(0.58-0.98)$ & 0.04 & $0.76(0.53-1.09)$ & 0.13 \\
\hline
\end{tabular}

PPARG

rs1801282

$\begin{array}{ll}C C & 548 \\ C G & 207 \\ \text { GG } & 14 \\ C G \operatorname{or} G G & 221\end{array}$

511

$167 \quad 0.87(0.68-1.10) \quad 0.23$

$15 \quad 1.15(0.55-2.40) \quad 0.71$

$182 \quad 0.88(0.70-1.11) \quad 0.29$

IL1B

rs4848306

GG $\quad 246$

GA $\quad 373$

AA 151

GA or AA 524

IL1B

rs1143623

$\begin{array}{ll}\text { GG } & 401 \\ \text { GC } & 316 \\ \text { CC } & 55 \\ \text { GC or CC } & 371\end{array}$

ILIB

rs1143627

$\begin{array}{ll}\text { TT } & 340 \\ \text { TC } & 339 \\ \text { CC } & 97 \\ \text { TC or CC } & 436\end{array}$

ILIRN

rs4251961

$\begin{array}{ll}\text { TT } & 298 \\ \text { TC } & 360 \\ \text { CC } & 112 \\ \text { TC or CC } & 472\end{array}$

IL4R

rs1805010

$\begin{array}{ll}\text { AA } & 209 \\ \text { AG } & 410 \\ \text { GG } & 157 \\ \text { AG or GG } & 567 \\ \text { L6 } & \\ \text { S10499563 } & \\ \text { TT } & 476 \\ \text { TC } & 259 \\ \text { CC } & 35 \\ \text { TC or CC } & 294\end{array}$

352

$125 \quad 0.95(0.70-1.28) \quad 0.72$

$477 \quad 1.04(0.83-1.30) \quad 0.72$

365

$1.04(0.69-1.56) \quad 0.85$

$330 \quad 0.98(0.80-1.20) \quad 0.83$

305

$305 \quad 1.00(0.81-1.25) \quad 0.98$

$86 \quad 0.99(0.71-1.37) \quad 0.94$

$391 \quad 1.00(0.81-1.23) \quad 1.00$

247

$324 \quad 1.09(0.87-1.36) \quad 0.47$

$429 \quad 1.10(0.89-1.36) \quad 0.40$

201

317

133

$0.80(0.63-1.02) \quad 0.08$

$0.88(0.65-1.19) \quad 0.41$

$450 \quad 0.83(0.66-1.04) \quad 0.10$

0.10

439

$225 \quad 0.94(0.76-1.17) \quad 0.60$

$26 \quad 0.81(0.48-1.36) \quad 0.42$

$251 \quad 0.93(0.75-1.14) \quad 0.48$
$1.04(0.83-1.32)$

0.71

$1.05(0.76-1.46) \quad 0.76$

$1.05(0.84-1.30) \quad 0.68$

0.07

$0.73(0.52-1.02)$

0.07

$0.91(0.67-1.24) \quad 0.55$

$0.87(0.58-1.33)$

0.53

$0.83(0.65-1.05) \quad 0.12$

$0.77(0.56-1.06)$

0.11

$0.94(0.75-1.18) \quad 0.60$

$0.77(0.57-1.05)$

0.10

$0.72(0.42-1.25)$

0.24

$0.80(0.39-1.63)$

0.53

$0.92(0.74-1.14) \quad 0.43$

$0.77(0.57-1.04)$

0.09 
Table 1 Odds ratios (OR) and 95\% confidence interval (95Cl) for genotypes studied among healthy controls and patients with ankylosing spondylitis (AS) (Continued)

\begin{tabular}{|c|c|c|c|c|c|c|c|c|}
\hline \multirow{2}{*}{$\begin{array}{l}\text { Gene } \\
\text { rs-number }\end{array}$} & \multirow[t]{2}{*}{ Healthy controls } & \multirow[t]{2}{*}{ AS } & \multicolumn{2}{|l|}{ Unadjusted } & \multicolumn{2}{|c|}{ Adjusted, age \& sex } & \multicolumn{2}{|c|}{ Adjusted, age, sex \& smoking } \\
\hline & & & $\mathrm{OR}(95 \mathrm{Cl})$ & p & OR $(95 \mathrm{Cl})$ & $p$ & OR $(95 \mathrm{Cl})$ & p \\
\hline \multicolumn{9}{|l|}{$\begin{array}{l}\text { IL6R } \\
\text { rs4537545 }\end{array}$} \\
\hline $\mathrm{CC}$ & 289 & 247 & & & & & & \\
\hline$C T$ & 369 & 324 & $1.03(0.82-1.29)$ & 0.82 & $1.05(0.83-1.32)$ & 0.71 & $1.07(0.79-1.47)$ & 0.65 \\
\hline$\pi$ & 117 & 113 & $1.13(0.83-1.54)$ & 0.44 & $1.18(0.86-1.63)$ & 0.30 & $1.17(0.76-1.79)$ & 0.48 \\
\hline $\mathrm{CT}$ or $\mathrm{TT}$ & 486 & 437 & $1.05(0.85-1.30)$ & 0.64 & $1.08(0.86-1.34)$ & 0.51 & $1.09(0.81-1.47)$ & 0.55 \\
\hline \multicolumn{9}{|l|}{$\begin{array}{l}\text { IL10 } \\
\text { rs1800872 }\end{array}$} \\
\hline CC & 482 & 408 & & & & & & \\
\hline CA & 258 & 225 & $1.03(0.83-1.29)$ & 0.79 & $1.01(0.80-1.27)$ & 0.94 & $0.93(0.68-1.26)$ & 0.63 \\
\hline $\mathrm{AA}$ & 35 & 42 & $1.42(0.89-2.26)$ & 0.14 & $1.35(0.83-2.18)$ & 0.22 & $1.47(0.79-2.73)$ & 0.22 \\
\hline CA or $A A$ & 293 & 267 & $1.08(0.87-1.33)$ & 0.50 & $1.05(0.84-1.30)$ & 0.67 & $0.99(0.74-1.33)$ & 0.95 \\
\hline \multicolumn{9}{|l|}{$\begin{array}{l}\text { IL10 } \\
\text { rs3024505 }\end{array}$} \\
\hline $\mathrm{CC}$ & 518 & 467 & & & & & & \\
\hline$C T$ & 221 & 200 & $1.00(0.80-1.26)$ & 0.97 & $1.01(0.80-1.28)$ & 0.95 & $1.19(0.87-1.61)$ & 0.28 \\
\hline$\pi$ & 22 & 24 & $1.21(0.67-2.19)$ & 0.53 & $1.32(0.72-2.42)$ & 0.37 & $1.80(0.79-4.12)$ & 0.16 \\
\hline $\mathrm{CT}$ or $\mathrm{TT}$ & 243 & 224 & $1.02(0.82-1.27)$ & 0.84 & $1.04(0.83-1.30)$ & 0.76 & $1.23(0.92-1.66)$ & 0.17 \\
\hline \multicolumn{9}{|l|}{$\begin{array}{l}\text { IL12B } \\
\text { rs3212217 }\end{array}$} \\
\hline GG & 499 & 460 & & & & & & \\
\hline GC & 235 & 200 & $0.92(0.74-1.16)$ & 0.49 & $0.95(0.75-1.19)$ & 0.64 & $0.94(0.69-1.29)$ & 0.72 \\
\hline $\mathrm{CC}$ & 25 & 21 & $0.91(0.50-1.65)$ & 0.76 & $0.94(0.51-1.72)$ & 0.84 & $0.57(0.23-1.41)$ & 0.22 \\
\hline GC or CC & 260 & 221 & $0.92(0.74-1.15)$ & 0.47 & $0.95(0.76-1.19)$ & 0.63 & $0.91(0.67-1.23)$ & 0.53 \\
\hline \multicolumn{9}{|l|}{$\begin{array}{l}\text { IL } 12 B \\
\text { rs6887695 }\end{array}$} \\
\hline GG & 385 & 324 & & & & & & \\
\hline GC & 293 & 301 & $1.22(0.98-1.52)$ & 0.07 & $1.24(0.99-1.55)$ & 0.06 & $1.31(0.97-1.77)$ & 0.07 \\
\hline $\mathrm{CC}$ & 72 & 70 & $1.16(0.81-1.66)$ & 0.43 & $1.16(0.80-1.69)$ & 0.43 & $0.98(0.59-1.61)$ & 0.94 \\
\hline GC or CC & 365 & 371 & $1.21(0.98-1.49)$ & 0.07 & $1.22(0.99-1.51)$ & 0.06 & $1.24(0.93-1.64)$ & 0.14 \\
\hline \multicolumn{9}{|l|}{$\begin{array}{l}\text { IL12RB1 } \\
\text { rs401502 }\end{array}$} \\
\hline CC & 360 & 304 & & & & & & \\
\hline$C G$ & 303 & 311 & $1.22(0.98-1.51)$ & 0.08 & $1.21(0.96-1.51)$ & 0.10 & $1.19(0.88-1.61)$ & 0.26 \\
\hline GG & 87 & 70 & $0.95(0.67-1.35)$ & 0.79 & $0.97(0.68-1.39)$ & 0.87 & $1.18(0.74-1.88)$ & 0.48 \\
\hline CG or GG & 390 & 381 & $1.16(0.94-1.42)$ & 0.17 & $1.15(0.93-1.43)$ & 0.19 & $1.19(0.89-1.58)$ & 0.24 \\
\hline \multicolumn{9}{|l|}{$\begin{array}{l}\text { IL17A } \\
\text { rs2275913 }\end{array}$} \\
\hline GG & 340 & 307 & & & & & & \\
\hline GA & 336 & 301 & $0.99(0.80-1.24)$ & 0.94 & $0.98(0.79-1.23)$ & 0.89 & $0.90(0.67-1.22)$ & 0.51 \\
\hline $\mathrm{AA}$ & 95 & 84 & $0.98(0.70-1.36)$ & 0.90 & $1.00(0.71-1.40)$ & 0.98 & $1.00(0.63-1.57)$ & 0.99 \\
\hline GA or $\mathrm{AA}$ & 431 & 385 & $0.99(0.80-1.22)$ & 0.92 & $0.99(0.80-1.22)$ & 0.89 & $0.92(0.69-1.22)$ & 0.57 \\
\hline \multicolumn{9}{|l|}{$\begin{array}{l}\text { IL18 } \\
\text { rs187238 }\end{array}$} \\
\hline GG & 387 & 380 & & & & & & \\
\hline
\end{tabular}


Table 1 Odds ratios (OR) and 95\% confidence interval (95Cl) for genotypes studied among healthy controls and patients with ankylosing spondylitis (AS) (Continued)

\begin{tabular}{|c|c|c|c|c|c|c|c|c|}
\hline \multirow{2}{*}{$\begin{array}{l}\text { Gene } \\
\text { rs-number }\end{array}$} & \multirow[t]{2}{*}{ Healthy controls } & \multirow[t]{2}{*}{ AS } & \multicolumn{2}{|l|}{ Unadjusted } & \multicolumn{2}{|c|}{ Adjusted, age \& sex } & \multicolumn{2}{|c|}{ Adjusted, age, sex \& smoking } \\
\hline & & & $\mathrm{OR}(95 \mathrm{Cl})$ & $p$ & $\mathrm{OR}(95 \mathrm{Cl})$ & $p$ & OR $(95 \mathrm{Cl})$ & $p$ \\
\hline GC & 312 & 259 & $0.85(0.68-1.05)$ & 0.13 & $0.83(0.66-1.03)$ & 0.09 & $0.74(0.55-1.00)$ & 0.049 \\
\hline CC & 64 & 41 & $0.65(0.43-0.99)$ & 0.04 & $0.69(0.45-1.06)$ & 0.09 & $0.58(0.32-1.04)$ & 0.07 \\
\hline GC or $C C$ & 376 & 300 & $0.81(0.66-1.00)$ & 0.0499 & $0.80(0.65-0.99)$ & 0.04 & $0.71(0.53-0.95)$ & 0.02 \\
\hline
\end{tabular}

1418

rs1946518

$\begin{array}{ll}\text { GG } & 282 \\ \text { GT } & 363 \\ \text { TT } & 113 \\ \text { GT or TT } & 476 \\ \text { IL23R } & \\ \text { rS } 11209026\end{array}$

259

GG

GA $\quad 89$

AA 5

GA or AA 94

IFNG

rs2430561

$\begin{array}{ll}\Pi \pi & 199 \\ \text { TA } & 398 \\ \text { AA } & 161\end{array}$

TA or AA 559

IFNGR1

rs2234711

$\begin{array}{ll}\text { TT } & 290 \\ \text { TC } & 361 \\ \text { CC } & 119 \\ \text { TC or CC } & 480\end{array}$

IFNGR2

rs8126756

$\begin{array}{ll}\text { TT } & 55 \\ \text { TC } & 168 \\ \text { CC } & 18 \\ \text { TC or CC } & 186\end{array}$

IFNGR2

rs17882748

$\begin{array}{ll}\text { CC } & 199 \\ \text { CT } & 391 \\ \text { TT } & 153 \\ \text { CT or TT } & 544 \\ \text { TBX21 } & \\ \text { IS17250932 } & \\ \text { TT } & 526 \\ \text { TC } & 210 \\ \text { CC } & 32 \\ \text { TC or CC } & 242\end{array}$

181

$398-369$

$369 \quad 1.02(0.80-1.30) \quad 0.88$

$139 \quad 0.95(0.70-1.29) \quad 0.74$

$508 \quad 1.00(0.79-1.26) \quad 0.99$

$\begin{array}{lll}329 & 0.99(0.79-1.24) & 0.91 \\ 97 & 0.93(0.68-1.29) & 0.68 \\ 426 & 0.97(0.79-1.21) & 0.81\end{array}$

$0.96(0.76-1.21)$

0.71

$0.89(0.65-1.21)$

0.45

$0.95(0.68-1.31) \quad 0.74$

$0.80(0.51-1.24)$

0.32

$0.96(0.77-1.19) \quad 0.68$

$0.86(0.64-1.16)$

0.32

59

290

\section{2}

$348 \quad 1.20(0.96-1.51) \quad 0.11$

$1.20(0.95-1.51) \quad 0.12$

$1.15(0.84-1.57)$

0.40

$108 \quad 1.13(0.83-1.55) \quad 0.43$

$1.09(0.79-1.50) \quad 0.60$

$1.11(0.72-1.70)$

0.65

$456 \quad 1.19(0.96-1.47) \quad 0.12$

$1.17(0.94-1.46) \quad 0.16$

$1.14(0.84-1.53)$

0.40

553

\section{2}

$130 \quad 0.82(0.63-1.06) \quad 0.13$

$0.83(0.64-1.09) \quad 0.18$

$0.86(0.60-1.24)$

0.42

$0.69(0.32-1.49) \quad 0.35$

$0.53(0.18-1.54) \quad 0.24$

$12 \quad 0.71(0.34-1.48) \quad 0.36$

$0.82(0.64-1.06) \quad 0.13$

$0.83(0.59-1.17)$

0.28

$142 \quad 0.81(0.63-1.04) \quad 0.09$

173

$341 \quad 1.00(0.78-1.29) \quad 0.98$

$1.00(0.77-1.30) \quad 0.99$

$\begin{array}{ll}1.01(0.71-1.42) & 0.97 \\ 1.16(0.77-1.73) & 0.48 \\ 1.05(0.76-1.45) & 0.76\end{array}$

$515 \quad 1.09(0.86-1.38) \quad 0.48$

$1.31(0.97-1.78) \quad 0.08$

$1.09(0.86-1.39) \quad 0.48$

TC or CC 242

497

$179 \quad 0.90(0.71-1.14) \quad 0.39$

$19 \quad 0.63(0.35-1.12) \quad 0.12$

$0.94(0.74-1.19) \quad 0.61$

$0.84(0.60-1.17)$

0.30

$198 \quad 0.87(0.69-1.08) \quad 0.21$

$0.66(0.36-1.19) \quad 0.17$

$0.37(0.14-0.98)$

0.046

$0.90(0.72-1.14) \quad 0.39$

$0.78(0.56-1.07)$

0.12 
Table 1 Odds ratios (OR) and 95\% confidence interval (95Cl) for genotypes studied among healthy controls and patients with ankylosing spondylitis (AS) (Continued)

\begin{tabular}{|c|c|c|c|c|c|c|c|c|}
\hline \multirow{2}{*}{$\begin{array}{l}\text { Gene } \\
\text { rs-number }\end{array}$} & \multirow[t]{2}{*}{ Healthy controls } & \multirow[t]{2}{*}{ AS } & \multicolumn{2}{|l|}{ Unadjusted } & \multicolumn{2}{|c|}{ Adjusted, age \& sex } & \multicolumn{2}{|c|}{ Adjusted, age, sex \& smoking } \\
\hline & & & OR $(95 \mathrm{Cl})$ & p & OR $(95 \mathrm{Cl})$ & $p$ & OR $(95 \mathrm{Cl})$ & $p$ \\
\hline \multicolumn{9}{|l|}{$\begin{array}{l}\text { NLRP1 } \\
\text { rs2670660 }\end{array}$} \\
\hline AA & 222 & 202 & & & & & & \\
\hline$A G$ & 390 & 328 & $0.92(0.73-1.18)$ & 0.52 & $0.96(0.75-1.23)$ & 0.73 & $1.12(0.80-1.56)$ & 0.52 \\
\hline GG & 154 & 154 & $1.10(0.82-1.47)$ & 0.53 & $1.11(0.82-1.49)$ & 0.51 & $1.12(0.75-1.67)$ & 0.59 \\
\hline$A G$ or $G G$ & 544 & 482 & $0.97(0.78-1.22)$ & 0.82 & $1.00(0.79-1.26)$ & 0.98 & $1.11(0.81-1.52)$ & 0.50 \\
\hline \multicolumn{9}{|l|}{$\begin{array}{l}\text { NLRP1 } \\
\text { rs878329 }\end{array}$} \\
\hline GG & 217 & 206 & & & & & & \\
\hline GC & 394 & 333 & $0.89(0.70-1.13)$ & 0.34 & $0.89(0.69-1.14)$ & 0.35 & $0.99(0.71-1.38)$ & 0.93 \\
\hline CC & 155 & 155 & $1.05(0.79-1.41)$ & 0.73 & $1.05(0.78-1.41)$ & 0.75 & $1.03(0.69-1.54)$ & 0.90 \\
\hline GC or CC & 549 & 488 & $0.94(0.75-1.17)$ & 0.57 & $0.93(0.74-1.18)$ & 0.56 & $1.00(0.73-1.36)$ & 0.98 \\
\hline \multicolumn{9}{|l|}{$\begin{array}{l}\text { NLRP3 } \\
\text { rs10754558 }\end{array}$} \\
\hline CC & 294 & 248 & & & & & & \\
\hline CG & 355 & 324 & $1.08(0.86-1.36)$ & 0.50 & $1.06(0.84-1.34)$ & 0.61 & $1.10(0.81-1.51)$ & 0.54 \\
\hline GG & 111 & 116 & $1.24(0.91-1.69)$ & 0.18 & $1.25(0.91-1.71)$ & 0.17 & $1.11(0.71-1.72)$ & 0.65 \\
\hline CG or GG & 466 & 440 & $1.12(0.90-1.39)$ & 0.30 & $1.11(0.89-1.38)$ & 0.36 & $1.11(0.82-1.49)$ & 0.51 \\
\hline \multicolumn{9}{|l|}{$\begin{array}{l}\text { NLRP3 } \\
\text { rs4612666 }\end{array}$} \\
\hline CC & 435 & 360 & & & & & & \\
\hline CT & 280 & 277 & $1.20(0.96-1.49)$ & 0.11 & $1.23(0.99-1.54)$ & 0.07 & $1.28(0.95-1.72)$ & 0.10 \\
\hline$\pi$ & 53 & 48 & $1.09(0.72-1.66)$ & 0.67 & 1.19 (0.78-1.82) & 0.41 & 1.07 (0.59-1.94) & 0.82 \\
\hline $\mathrm{CT}$ or TT & 333 & 325 & $1.18(0.96-1.45)$ & 0.12 & $1.23(0.99-1.52)$ & 0.06 & $1.24(0.94-1.65)$ & 0.13 \\
\hline \multicolumn{9}{|l|}{$\begin{array}{l}\text { CARD8 } \\
\text { rs2043211 }\end{array}$} \\
\hline AA & 321 & 298 & & & & & & \\
\hline AT & 342 & 316 & $1.00(0.80-1.24)$ & 0.97 & $0.98(0.79-1.23)$ & 0.89 & $0.90(0.67-1.22)$ & 0.50 \\
\hline$\pi$ & 94 & 78 & $0.89(0.64-1.25)$ & 0.52 & $0.89(0.63-1.26)$ & 0.50 & $0.91(0.57-1.44)$ & 0.68 \\
\hline AT or TT & 436 & 394 & $0.97(0.79-1.20)$ & 0.80 & $0.96(0.78-1.19)$ & 0.72 & $0.90(0.67-1.19)$ & 0.45 \\
\hline \multicolumn{9}{|l|}{$\begin{array}{l}\text { JAK2 } \\
\text { rs } 12343867\end{array}$} \\
\hline$\pi$ & 398 & 358 & & & & & & \\
\hline $\mathrm{TC}$ & 299 & 263 & $0.98(0.79-1.22)$ & 0.84 & $0.96(0.76-1.20)$ & 0.69 & $0.82(0.61-1.12)$ & 0.21 \\
\hline CC & 61 & 65 & $1.18(0.81-1.73)$ & 0.38 & $1.11(0.75-1.63)$ & 0.61 & $1.03(0.62-1.71)$ & 0.91 \\
\hline TC or CC & 360 & 328 & $1.01(0.82-1.25)$ & 0.90 & $0.98(0.79-1.21)$ & 0.86 & $0.86(0.64-1.14)$ & 0.29 \\
\hline
\end{tabular}

\section{Power calculation}

The Genetic Power Calculator was utilized for power analysis of discrete traits (http://zzz.bwh.harvard.edu/ gpc/cc2.html). The lowest minor allele frequency (MAF) of the studied SNPs was 0.10. The 'high-risk allele frequency' was set to 0.10 , the 'prevalence' was set to 0.0018 [18], D-prime was set to 1 , type I error rate was set to 0.05 and number of cases and control:case ratio was 795:709. This cohort study had more than $80 \%$ chance of detecting a dominant effect with an odds ratio (OR) of 1.4 for AS.

\section{Statistical analysis}

Logistic regression was used to compare genotype distributions among patients with AS versus healthy controls. Crude odds ratio, odds ratio adjusted for age and sex, and odds ratio adjusted for age, sex, and smoking status were assessed (Table 1). A chi-square test was 


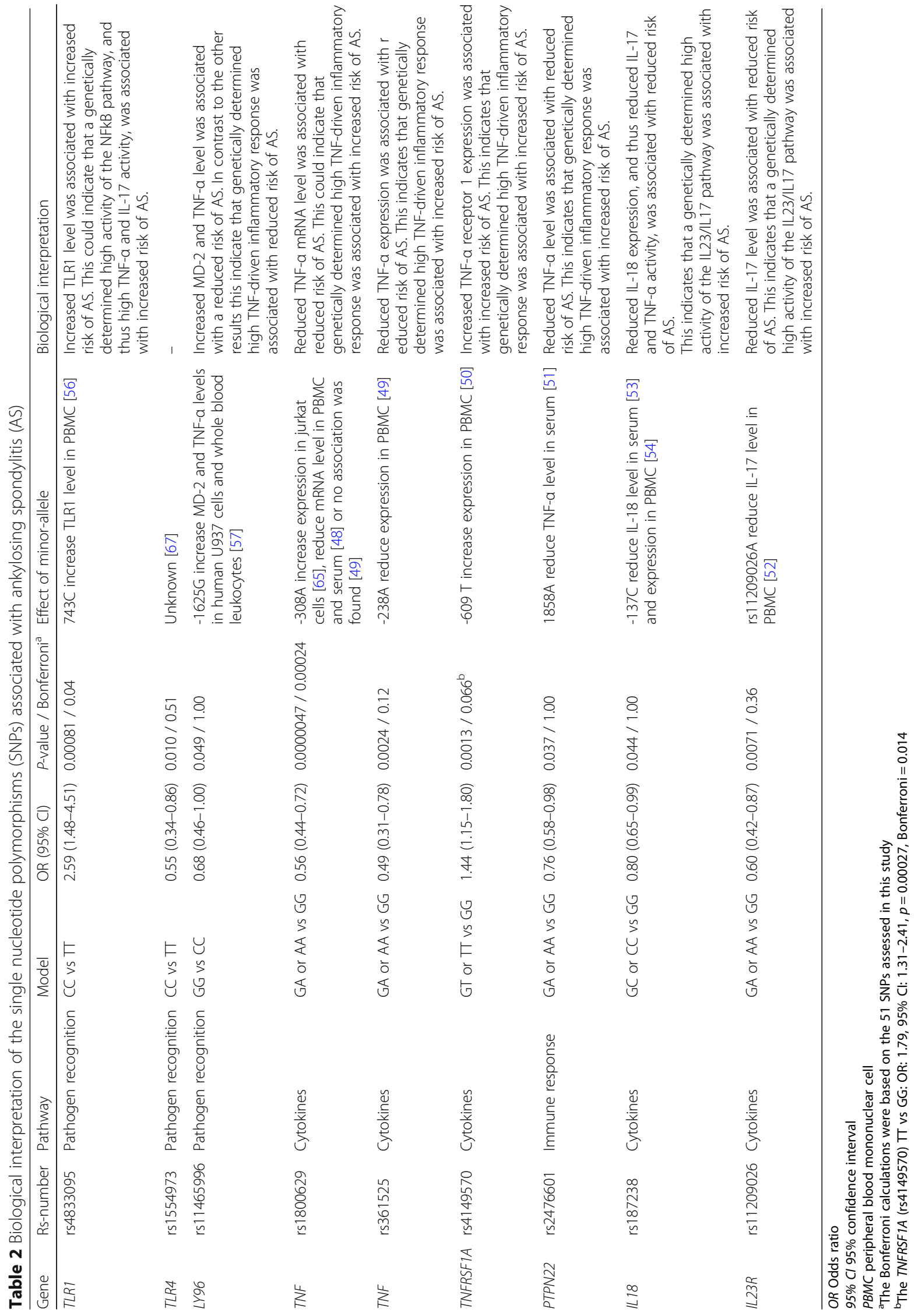


Table 3 Association of the TLR2 haplotype combinations and risk of ankylosing spondylitis (AS). The haplotype combinations in TLR2 described $93 \%$ of the genotypes observed

\begin{tabular}{|c|c|c|c|c|c|c|c|c|}
\hline \multirow{2}{*}{$\begin{array}{l}\text { Haplotype } \\
\text { combinations }\end{array}$} & \multicolumn{3}{|l|}{ Haplotypes } & \multirow[t]{2}{*}{$\mathrm{N}_{\mathrm{AS}}(\%)$} & \multirow[t]{2}{*}{$\mathrm{N}_{\text {Control }}(\%)$} & \multirow[t]{2}{*}{$\mathrm{OR}^{\mathrm{a}}$} & \multirow[t]{2}{*}{$(95 \% \mathrm{Cl})$} & \multirow[t]{2}{*}{$P$-value } \\
\hline & rs4696480 A>T & rs11938228 C > A & rs3804099 T>C $C^{b}$ & & & & & \\
\hline 11 & $\mathrm{~T}: \mathrm{T}$ & $A: A$ & $\mathrm{~T}: \mathrm{T}$ & $69(11)$ & $76(10)$ & 1.00 & - & - \\
\hline 22 & $A: A$ & $C: C$ & $C: C$ & $72(11)$ & $74(10)$ & 1.07 & $0.68-1.70$ & 0.82 \\
\hline 33 & $A: A$ & $C: C$ & $\mathrm{~T}: \mathrm{T}$ & $28(4)$ & $34(5)$ & 0.91 & $0.50-1.65$ & 0.76 \\
\hline 44 & $\mathrm{~T}: \mathrm{T}$ & $C: C$ & $C: C$ & $14(2)$ & $10(1)$ & 1.52 & $0.64-3.70$ & 0.38 \\
\hline 12 & $\mathrm{~T}: \mathrm{A}$ & $C: A$ & C:T & $158(24)$ & $197(27)$ & 0.88 & $0.60-1.30$ & 0.55 \\
\hline 13 & $\mathrm{~T}: \mathrm{A}$ & $C: A$ & $\mathrm{~T}: \mathrm{T}$ & $76(12)$ & $103(14)$ & 0.81 & $0.52-1.26$ & 0.37 \\
\hline 14 & $\mathrm{~T}: \mathrm{T}$ & $C: A$ & C:T & $59(9)$ & $49(7)$ & 1.33 & $0.80-2.19$ & 0.31 \\
\hline 23 & $A: A$ & $C: C$ & $C: T$ & $77(12)$ & $89(12)$ & 0.95 & $0.61-1.49$ & 0.91 \\
\hline 24 & $\mathrm{~T}: \mathrm{A}$ & $C: C$ & $C: C$ & $52(8)$ & $55(8)$ & 1.04 & $0.63-1.72$ & 0.90 \\
\hline 34 & $\mathrm{~T}: \mathrm{A}$ & $C: C$ & $C: T$ & $51(8)$ & $44(6)$ & 1.28 & $0.76-2.14$ & 0.43 \\
\hline
\end{tabular}

OR Odds ratio

${ }^{a}$ OR was calculated for each haplotype combination by using the haplotype 11 as reference group

${ }^{\mathrm{b}}$ The variant allele of rs3804099T T > C has been shown to decrease TNF- $a$, IL-1 $\beta$ \& IL-6 level [68]

used to test for deviation from Hardy-Weinberg equilibrium in the healthy controls and for haplotype analysis (Tables 3, 4, 5 and 6).

Statistical analyses were performed using STATA version 15 (StataCorp LP, College Station, TX, USA).

\section{Results}

\section{Study population}

Among the patients with AS the median age was 32 years (SD: 11.5$)$ and 68\% (483/709) were males. The healthy controls had a median age of 43 years (SD: 11.5 ) and $52 \%$ (411/384) were males. Among the patients 37\% (118/323), $23 \%(73 / 323)$, and $41 \%(132 / 323)$ and among the controls 26\% (207/788), 24\% (189/788), and 50\% (392/788) were current smokers, former smokers and never smokers, respectively. HLA-B27 staus was available for 498 patients of which $83 \%$ (411/498) were positive. Sixty percent (427/ 709) of the patients were treated with anti-TNF.

The genotype distributions among the healthy controls deviated from Hardy-Weinberg equilibrium for TLR1 $(743 \mathrm{~T}>\mathrm{C}(\mathrm{rs} 4833095))(p=0.03), \operatorname{TLR} 2(-16,934 \mathrm{~A}>\mathrm{T}$ $(\mathrm{rs} 4696480))(p=0.02)$, TLR4 $(\mathrm{rs} 1554973 \mathrm{~T}>\mathrm{C})(p=0.03)$, TLR9 $(1174 \mathrm{G}>\mathrm{A}(\mathrm{rs} 352139))(p=0.02)$ and TGFB1 $(-509$ $\mathrm{C}>\mathrm{T}(\mathrm{rs} 1800469))(p=0.02)$. After correction for multiple testing, all SNPs studied were in Hardy-Weinberg equilibrium.

\section{Polymorphisms associated with susceptibility of AS}

In the age and sex adjusted analysis, the homozygous variant genotype of TLR1 $743 \mathrm{~T}>\mathrm{C}$ (rs4833095) (OR: 2.59,

Table 4 Association between TLR4 haplotype combinations and risk of ankylosing spondylitis (AS). The haplotype combinations in TLR4 described $94 \%$ of the genotypes observed

\begin{tabular}{|c|c|c|c|c|c|c|c|c|}
\hline \multirow{2}{*}{$\begin{array}{l}\text { Haplotype } \\
\text { combinations }\end{array}$} & \multicolumn{3}{|l|}{ Haplotypes } & \multirow[t]{2}{*}{$\mathrm{N}_{\mathrm{AS}}(\%)$} & \multirow[t]{2}{*}{$\mathrm{N}_{\text {Control }}(\%)$} & \multirow[t]{2}{*}{$O R^{a}$} & \multirow[t]{2}{*}{$(95 \% \mathrm{Cl})$} & \multirow[t]{2}{*}{$P$-value } \\
\hline & $\begin{array}{l}r s 12377632 \\
T>C\end{array}$ & $\begin{array}{l}\text { rs } 1554973 \\
T>C\end{array}$ & $\begin{array}{l}\mathrm{rs} 5030728 \\
\mathrm{G}>\mathrm{A}\end{array}$ & & & & & \\
\hline 11 & $C: C$ & $\mathrm{~T}: \mathrm{T}$ & G:G & 95 (14) & $101(14)$ & 1.00 & - & - \\
\hline 22 & $\mathrm{~T}: \mathrm{T}$ & $\mathrm{T}: \mathrm{T}$ & $A: A$ & $69(10)$ & $74(10)$ & 0.99 & $0.64-1.53$ & 1.00 \\
\hline 33 & $\mathrm{~T}: \mathrm{T}$ & $C: C$ & G:G & $29(4)$ & $57(8)$ & 0.54 & $0.32-0.92$ & 0.03 \\
\hline 44 & $\mathrm{~T}: \mathrm{T}$ & $\mathrm{T}: \mathrm{T}$ & G:G & $3(0)$ & $5(1)$ & 0.64 & $0.15-2.74$ & 0.72 \\
\hline 12 & $\mathrm{~T}: \mathrm{C}$ & $\mathrm{T}: \mathrm{T}$ & $\mathrm{G}: \mathrm{A}$ & 154 (23) & $188(25)$ & 0.87 & $0.61-1.24$ & 0.47 \\
\hline 13 & $\mathrm{~T}: \mathrm{C}$ & $\mathrm{T}: \mathrm{C}$ & $\mathrm{G}: \mathrm{G}$ & 126 (19) & 129 (17) & 1.04 & $0.72-1.51$ & 0.85 \\
\hline 14 & $\mathrm{~T}: \mathrm{C}$ & $\mathrm{T}: \mathrm{T}$ & G:G & $30(5)$ & $32(4)$ & 1.00 & $0.56-1.77$ & 1.00 \\
\hline 23 & $\mathrm{~T}: \mathrm{T}$ & $\mathrm{T}: \mathrm{C}$ & $\mathrm{G}: \mathrm{A}$ & $99(15)$ & $106(14)$ & 0.99 & $0.67-1.47$ & 1.00 \\
\hline 24 & $\mathrm{~T}: \mathrm{T}$ & $\mathrm{T}: \mathrm{T}$ & G:A & $31(5)$ & $24(3)$ & 1.37 & $0.75-2.51$ & 0.36 \\
\hline 34 & $\mathrm{~T}: \mathrm{T}$ & $\mathrm{T}: \mathrm{C}$ & $\mathrm{G}: \mathrm{G}$ & $28(4)$ & $26(4)$ & 1.14 & $0.63-2.09$ & 0.76 \\
\hline
\end{tabular}


Table 5 Association between IL1B haplotype combinations and risk of ankylosing spondylitis (AS). The haplotype combinations in ILIB described $97 \%$ of the genotypes observed

\begin{tabular}{|c|c|c|c|c|c|c|c|c|}
\hline \multirow{2}{*}{$\begin{array}{l}\text { Haplotype } \\
\text { combinations }\end{array}$} & \multicolumn{3}{|l|}{ Haplotypes } & \multirow[t]{2}{*}{$N_{A S}(\%)$} & \multirow[t]{2}{*}{$\mathrm{N}_{\text {Control }}(\%)$} & \multirow[t]{2}{*}{$\mathrm{OR}^{\mathrm{a}}$} & \multirow[t]{2}{*}{$(95 \% \mathrm{Cl})$} & \multirow[t]{2}{*}{$P$-value } \\
\hline & $\begin{array}{l}\text { rs4848306 } \\
-3737 G>A[69,70]\end{array}$ & $\begin{array}{l}\text { rs1143623 } \\
-1464 G>C[69,71]\end{array}$ & $\begin{array}{l}\text { rs } 1143627 \\
-31 \mathrm{~T}>\mathrm{C}[69,71,72]\end{array}$ & & & & & \\
\hline 11 & $A: A$ & G:G & $\mathrm{T}: \mathrm{T}$ & 125 (18) & $148(20)$ & 1.00 & - & - \\
\hline 22 & G:G & $\mathrm{C}: \mathrm{C}$ & $\mathrm{C}: \mathrm{C}$ & $52(8)$ & $54(7)$ & 1.14 & $0.73-1.79$ & 0.65 \\
\hline 33 & G:G & G:G & $\mathrm{T}: \mathrm{T}$ & $32(5)$ & $41(5)$ & 0.92 & $0.55-1.55$ & 0.79 \\
\hline 44 & G:G & G:G & $C: C$ & $5(1)$ & $3(0)$ & 1.97 & $0.46-8.42$ & 0.48 \\
\hline 12 & $A: G$ & $\mathrm{G}: \mathrm{C}$ & $\mathrm{T}: \mathrm{C}$ & $163(24)$ & $185(24)$ & 1.04 & $0.76-1.43$ & 0.81 \\
\hline 13 & $A: G$ & G:G & $\mathrm{T}: \mathrm{T}$ & $141(20)$ & 147 (19) & 1.14 & $0.82-1.58$ & 0.50 \\
\hline 14 & $A: G$ & G:G & $\mathrm{T}: \mathrm{C}$ & $44(6)$ & $38(5)$ & 1.37 & $0.84-2.25$ & 0.26 \\
\hline 23 & G:G & $C: G$ & $C: T$ & $84(12)$ & $92(12)$ & 1.08 & $0.74-1.58$ & 0.70 \\
\hline 24 & G:G & $C: G$ & $C: C$ & $28(4)$ & $34(4)$ & 0.98 & $0.56-1.70$ & 1.00 \\
\hline 34 & G:G & G:G & $\mathrm{T}: \mathrm{C}$ & $14(2)$ & $16(2)$ & 1.04 & $0.49-2.21$ & 1.00 \\
\hline
\end{tabular}

\section{OR Odds ratio}

The variant allele of $-3737 \mathrm{G}>\mathrm{A}$ [69], $-1464 \mathrm{G}>\mathrm{C}[70]$ and $-31 \mathrm{~T}>\mathrm{C}[71,72]$ have been shown to decrease IL-1 $\beta$ level [69-72]

${ }^{a} \mathrm{OR}$ was calculated for each haplotype combination by using the haplotype 11 as reference group

95\% CI: $1.48-4.51, p=0.0008)$ and the combined homozygous and the heterozygous variant genotypes of TNFRSF1A -609 G > T (rs4149570) (OR: 1.44, 95\% CI: 1.15-1.80, $p=0.001)$ were associated with increased risk of AS. The homozygous variant genotype of TLR4 T>C (rs1554973) (OR: 0.55, 95\% CI: 0.34-0.86, $p=0.01$ ) and LY96-1625 C > G (rs11465996) (OR: 0.68, 95\% CI: $0.46-1.00, p=0.05)$, and the combined homozygous and the heterozygous variant genotypes of TNF -308 G>A (rs1800629) (OR: 0.56, 95\% CI: 0.44-0.72, $p=0.000005$ ), TNF -238 G > A (rs361525) (OR: 0.49, 95\% CI: 0.31-0.78, $p=0.002)$, PTPN22 $1858 \mathrm{G}>\mathrm{A}$ (rs2476601) (OR: 0.76, 95\% CI: 0.58-0.98, $p=0.04), I L 18-137 \mathrm{G}>\mathrm{C}(\mathrm{rs} 187238)$ (OR: 0.80, 95\% CI: 0.65-0.99, $p=0.04$ ), and IL23R G > A (rs11209026) (OR: 0.60, 95\% CI: 0.42-0.87, $p=0.01$ ) were associated with reduced risk of AS (Table 1).

After Bonferroni correction for multiple testing the homozygous variant genotype of TLR1 $743 \mathrm{~T}>\mathrm{C}$ (rs4 833095) (OR: 2.59, 95\% CI: 1.48-4.51, $p=0.04$ ) and TNFRSF1A -609 G > T (rs4149570) (OR: 1.79, 95\% CI:
1.31-2.41, $\mathrm{p}=0.01)$ were associated with increased risk of AS and the combined homozygous and the heterozygous variant genotypes of TNF $-308 \mathrm{G}>\mathrm{A}$ (rs1800629) (OR: 0.56, 95\% CI: $0.44-0.72, p=0.0002$ ) were associated with reduced risk of AS (Table 2).

SNPs associated with AS and the biological effect of the SNPs are summarized in Table 2.

\section{Haplotype analysis}

Haplotype analyses of TLR2, TLR4, IL1B and TNF are shown in Tables 3, 4, 5 and 6, respectively.

The TLR4 haplotype combination 33 (rs12377632TT, rs1554973CC and rs5030728GG) was associated with reduced risk of AS (OR: 0.54, 95\% CI: 0.32-0.92, $p=0.03$ ) compared to the haplotype combination 11 . In TNF all haplotype combinations were associated with reduced risk of AS compared to the haplotype combination 11 (rs361525GG and rs1800629GG).

No associations were found for haplotype combinations of TLR2 or IL1B.

Table 6 Association of the TNF haplotype combinations and risk of ankylosing spondylitis (AS). The haplotype combinations in TNF described $97 \%$ of the genotypes observed

\begin{tabular}{|c|c|c|c|c|c|c|c|}
\hline \multirow{2}{*}{$\begin{array}{l}\text { Haplotype } \\
\text { combinations }\end{array}$} & \multicolumn{2}{|l|}{ Haplotypes } & \multirow[t]{2}{*}{$\mathrm{N}_{\mathrm{AS}}(\%)$} & \multirow[t]{2}{*}{$\mathrm{N}_{\text {Control }}(\%)$} & \multirow[t]{2}{*}{$\mathrm{OR}^{\mathrm{a}}$} & \multirow[t]{2}{*}{$(95 \% \mathrm{Cl})$} & \multirow[t]{2}{*}{$P$-value } \\
\hline & $r s 361525 G>A^{b}$ & rs1800629 G>Ac & & & & & \\
\hline 11 & $\mathrm{G}: \mathrm{G}$ & $\mathrm{G}: G$ & $523(76)$ & $469(61)$ & 1.00 & - & - \\
\hline 22 & $\mathrm{G}: \mathrm{G}$ & $A: A$ & $9(1)$ & $25(3)$ & 0.32 & $(0.15-0.70)$ & 0.005 \\
\hline 12 & $\mathrm{G}: \mathrm{G}$ & $\mathrm{G}: \mathrm{A}$ & $125(18)$ & $210(28)$ & 0.53 & $(0.41-0.69)$ & $<0.0001$ \\
\hline 13 & $\mathrm{G}: \mathrm{A}$ & $\mathrm{G}: \mathrm{G}$ & $26(4)$ & $47(6)$ & 0.50 & $(0.30-0.81)$ & 0.007 \\
\hline 14 & $\mathrm{G}: \mathrm{A}$ & $\mathrm{G}: \mathrm{A}$ & $4(1)$ & $12(2)$ & 0.30 & $(0.10-0.93)$ & 0.05 \\
\hline
\end{tabular}

\section{OR Odds ratio}

${ }^{a}$ OR was calculated for each haplotype combination by using the haplotype 11 as reference group

${ }^{\mathrm{b}}$ The variant allele of TNF -238A rs361525A G > A has been shown to reduce expression of TNF-a [49]

'The variant allele of TNF -308A rs $1800629 \mathrm{G}>\mathrm{A}$ has been shown to reduce mRNA level [48] 


\section{Discussion}

In this case-control study, polymorphisms in a: the TNF- $\alpha$ (TNF (rs1800629 and rs361525), TNFRSF1A (rs4149570), and PTPN22 (rs2476601)), b: the IL23/IL17 (IL23R (rs11 209026), and IL18 (rs187238)), or c: the NFkB (TLR1 (rs4833095), TLR4 (rs1554973), and LY96 (rs11465996)) pathways were associated with risk of AS.

The found assocaitions for TNF (rs1800629) [19-22], TNFRSF1A (rs4149570) [23], and IL23R (rs11209026) [24-33] are in agreement with other case-control studies. Furthermore, Zhao et al. found that the variant allele of NLRP3 (rs4612666) was associated with increased risk of AS in Chinese patients [23]. In our study we found a trend for associations of the variant allele of NLRP3 (rs4612666) with increased risk of AS $(p=0.06)$. However, our results are in contrast to a meta-analysis of the PTPN22 (rs2476601) polymorphism that did not find an association with AS [34]. Finally, we identified novel risk loci in TNF (rs361525), IL18 (rs187238), TLR1 (rs48 33095), TLR4 (rs1554973), and LY96 (rs11465996) that need validation in independent cohorts.

Most of the SNPs assessed in our study have known biological effects thus allowing a biological interpretation of the observed associations based on increased or reduced gene activity as summarized in Table 2 [35-47]. The associations observed for the TNF (rs1800629 and rs361525) polymorphisms suggest that reduced TNF- $\alpha$ mRNA level and expression of TNF- $\alpha$ was associated with reduced risk of AS [48, 49]. This is supported by our haplotype analysis which also suggests that the variant alleles of TNF rs1800629 and rs361525 were associated with reduced risk of AS. Likewise, the associations observed for the TNFRSF1A (rs4149570) polymorphism indicates that increased expression of the TNF- $\alpha$ receptor 1 was associated with increased risk of AS [50]. Furthermore, the associations observed for the PTPN22 (rs2476601) polymorphism suggests that reduced TNF- $\alpha$ serum level was associated with reduced risk of AS [51]. Taken together, this suggests that genetically determined high activity of the TNF- $\alpha$ pathway was associated with increased risk of AS.

IL-17 is known to induce the production of many cytokines including TNF- $\alpha$ [6]. IL-18 is a pro-inflammatory cytokine known to enhance the production of IL-17, TNF- $\alpha$, and IL-1 $\beta$ [8]. In this study, the association observed for the IL23R (rs11209026) polymorphism suggests that reduced IL-17 serum level, and thus reduced TNF- $\alpha$ activity, was associated with reduced risk of AS [52]. Furthermore, the associations observed for the IL18 (rs187238) polymorphism indicates that reduced IL-18 expression, and thus reduced IL-17 and TNF- $\alpha$ activity, was associated with reduced risk of AS [53, 54]. The associations found in the IL23R (rs11209026) and the IL18 (rs187238) polymorphisms thus suggest that a genetically determined high activity of the IL23/IL17 pathway was associated with increased risk of AS. The two SNPs furthermore support that genetically determined high activity of the TNF- $\alpha$ pathway was associated with increased risk of AS. The observed associations between the polymorphisms in IL23R and IL18 and risk of AS are in line with previous studies pointing out the IL23/IL17 pathway as central to the pathophysiology of AS $[3,4,55]$.

This study also suggests that the NFkB pathway may be involved in the etiology of AS. The associations observed for the TLR1 (rs4833095) polymorphism suggests that increased TLR1 level was associated with increased risk of AS [56]. High level of TLR1 may lead to increased NFkB activation and thus increased TNF- $\alpha$ and IL-17 activity, which is in line with the other results. However, in contrast to the other results, the associations observed for the LY96 (rs11465996) polymorphism suggests that increased MD-2 (LY96) and TNF- $\alpha$ level was associated with a reduced risk of AS [57]. Finally, the TLR4 (rs1554973) polymorphism was associated with reduced risk of AS which was supported by the haplotype results (Table 4). The biological effect of the TLR4 (rs1554973) polymorphism is unknown, however, the result supports the notion that the NFkB pathway may be involved in the etiology of AS.

Both TNF- $\alpha$ [58] and interleukin-17 inhibitors [59] have been shown to reduce inflammation and improve symptoms in patients with AS [60]. Furthermore, increased levels of TNF- $\alpha$, IL-17, IL-23, IL-1 $\beta$, and IL-6 have been found in sera and synovial fluid from AS patients [61-64]. The genetic associations between AS and the polymorphisms in TLR1, TLR4, LY96, TNF, TNFRSF1A, $I L 18$, and IL23R found in this study, could potentially - in part - explain this altered cytokine milieu present in AS patients.

There are aspects of this study which should be interpreted with care. Conflicting results have been reported for the TNF (rs1800629) polymorphism [48, 49, 65]. Furthermore, the TNF polymorphisms, as well as the HLA-B27 locus, are located on chromosome 6 , and there is a risk that even a minor linkage disequilibrium could have confounded our results [2]. TLR1 (rs4833095), TLR2 (rs4696480), TLR4 (rs1554973), TLR9 (rs352139), and TGFB1 (rs1800469) were not in Hardy-Weinberg equilibrium among the healthy controls. Due to the number of polymorphisms analyzed this is probably a type II error. The polymorphisms do not deviate from Hardy-Weinberg equilibrium when corrected for multiple testing. We cannot exclude that some of our positive findings may be due to chance due to the obtained $p$-values and the number of statistical tests performed. When the results were corrected for multiple testing only the variant allele of TLR1 (rs4833095) and TNFRSF1A (rs4149570) were associated with increased risk of AS and the 
variant allele of TNF (rs1800629) was associated with reduced risk of AS.

A major strength of this study was that the cohort was rather large including 709 patients with AS and 795 healthy controls and the associations that we report were biologically plausible. Also, the validity of the diagnosis is expected to be high, since the patients were identified via a clinical database that the rheumatologist use for prospective monitoring of patients as part of routine care [66].

\section{Conclusions}

In conclusion, we replicated associations between AS and the polymorphism TNF (rs1800629), TNFRSF1A (rs4149570), and IL23R (rs11209026). Furthermore, we identified novel risk loci in TNF (rs361525), IL18 (rs18 7238), TLR1 (rs4833095), TLR4 (rs1554973), and LY96 (rs11465996) that need validation in independent cohorts. The results suggest that genetically determined high activity of the TNF- $\alpha$, IL23/IL17, and NFkB pathways increase the risk of AS.

\section{Acknowledgments}

We thank Ewa Kogutowska and Mette Errebo Rønne, Statens Serum Institut, for laboratory support; and Niels Steen Krogh, Zitelab Aps, Copenhagen, Denmark for database management. We also thank Department of Medicine, Viborg Regional Hospital, Denmark and OPEN (Odense Patient data Explorative Network), Odense University Hospital, Denmark for supporting this work. In memory of Niels Henrik Heegaard:

Co-author Niels H.H. Heegaard, Professor, MD, DMSc, DNatSc, died unexpectedly on September 26, 2017, at age 57. As director of the Department of Autoimmunology and Biomarkers, Statens Serum Institut, Copenhagen, Dr. Heegaard advanced research in autoimmunology and neurodegenerative disease. He had an extensive international research network and published more than 200 papers in scientific journals, focusing on biomarkers such as autoantibodies, microRNA, and microparticle proteins. He was a patient and unpretentious collaborator who always sought to highlight the work of other collaborators and co-workers. Dr. Heegaard was characterized by humor, kindness, and optimism. He is survived by his wife and 2 children.

\section{Funding}

This study was funded by the Danish Rheumatism Association (A1923, A3037, and A3570 - www. Gigtforeningen.dk) and Region of Southern Denmark's PhD Fund, 12/7725 (www. Regionsyddanmark.dk).

\section{Availability of data and materials}

The datasets used during the current study are available from the corresponding author on reasonable request.

\section{Authors' contributions}

JS, SB, UV, PSA, SBS, HL, NHH and VA designed the research study and PSA, ABB, MRA, IB, RBD, $H J H, B G$ and MLH collected the materials. JS and SB analysed the data and wrote the first draft. UV, PSA, SBS, ABB, MRA, IB, RBD, HJH, BG, MLH, HL and VA critically revised the manuscript. All authors agreed to be accountable for all aspects of the work and approved the final version of the manuscript.

\section{Ethics approval and consent to participate}

The study was conducted in accordance with the Declaration of Helsinki and was approved by the Regional Ethics Committees of Central (M20100153) and Southern (S-20120113) Denmark and the Danish Data Protection Agency of Central (RM: J. 2010-41-4719) and Southern (RSD: 2008-58-035) Denmark. For blood samples collected after routine TB screening, the Ethics Committees gave exemption from informed consent requirements because samples were taken as part of routine care and data were not identifiable. Written informed consent was obtained from patients donating blood samples at Frederiksberg Hospital as this involved collecting additional samples from patients.
Consent for publication

Not applicable.

\section{Competing interests}

$\checkmark A$ receives compensation as a consultant and for being member of an advisory board for MSD and Janssen. BG has recived research funding from AbbVie, Biogen, Pfizer. The other authors declare no conflicts of interest.

\section{Publisher's Note}

Springer Nature remains neutral with regard to jurisdictional claims in published maps and institutional affiliations.

\section{Author details}

${ }^{1}$ Institute of Regional Health Research, University of Southern Denmark, Odense, Denmark. ${ }^{2}$ Department of Autoimmunology and Biomarkers, Statens Serum Institut, Copenhagen, Denmark. ${ }^{3}$ Department of Rheumatology, Frederiksberg Hospital, Frederiksberg, Denmark. ${ }^{4}$ Department of Rheumatology, Skåne University Hospital, Lund, Sweden. ${ }^{5}$ Focused Research Unit for Molecular Diagnostic and Clinical Research, Hospital of Southern Jutland, Aabenraa, Denmark. ${ }^{6}$ Medical Department, Viborg Regional Hospital, Viborg, Denmark. ${ }^{7}$ National Research Centre for the Working Environment, Copenhagen, Denmark. ${ }^{8}$ Microbiology and Infection Control, Statens Serum Institut, Copenhagen, Denmark. 'Veterinary Disease Biology, University of Copenhagen, Copenhagen, Denmark. ${ }^{10}$ Institute of Molecular Medicine, University of Southern Denmark, Odense, Denmark. ${ }^{11}$ Department of Clinical Biochemistry, Herlev and Gentofte Hospital, Hellerup, Denmark.

${ }^{12}$ Department of Biochemistry, Hospital of Lillebaelt, Vejle, Denmark.

${ }^{13}$ Department of Clinical Microbiology, Slagelse Hospital, Slagelse, Denmark.

${ }^{14}$ Department of Clinical Medicine, Aarhus University, Aarhus, Denmark.

${ }^{15}$ Department of Respiratory Diseases B, Aarhus University Hospital, Aarhus, Denmark. ${ }^{16}$ Department of Rheumatology, Gentofte and Herlev Hospital, Hellerup, Denmark. ${ }^{17}$ The DANBIO Registry, Copenhagen Center for Arthritis Research, Center for Rheumatology and Spine Diseases, Rigshospitalet, Glostrup, Denmark. ${ }^{18}$ Department of Clinical Medicine, Faculty of Health and Medical Sciences, University of Copenhagen, Copenhagen, Denmark. ${ }^{19}$ Clinical Biochemistry, Clinical Institute, University of Southern Denmark, Odense, Denmark. ${ }^{20}$ OPEN Odense Patient Data Explorative Network, Odense University Hospital, Odense, Denmark.

Received: 17 May 2018 Accepted: 3 September 2018

Published online: 12 September 2018

\section{References}

1. Khan MA. Ankylosing spondylitis. In: Oxford University press; 2009. https:// global.oup.com/academic/product/ankylosing-spondylitis9780195368079?cc=dk\&lang=en\&\#

2. Brown MA, Kenna T, Wordsworth BP. Genetics of ankylosing spondylitis-insights into pathogenesis. Nat Rev Rheumatol. 2016;12(2):81-91.

3. Yago T, et al. IL-23 and Th17 disease in inflammatory arthritis. J Clin Med. 2017;6(9):E81

4. Raychaudhuri SP, Raychaudhuri SK. Mechanistic rationales for targeting interleukin-17A in spondyloarthritis. Arthritis Res Ther. 2017;19(1):51.

5. Verstrepen L, et al. TLR-4, IL-1R and TNF-R signaling to NF-kappaB: variations on a common theme. Cell Mol Life Sci. 2008;65(19):2964-78.

6. Hoeve MA, et al. Divergent effects of IL-12 and IL-23 on the production of IL-17 by human T cells. Eur J Immunol. 2006;36(3):661-70.

7. Aguilera M, Darby T, Melgar S. The complex role of inflammasomes in the pathogenesis of inflammatory bowel diseases - lessons learned from experimental models. Cytokine Growth Factor Rev. 2014;25(6):715-30.

8. Dinarello CA, et al. Interleukin-18 and IL-18 binding protein. Front Immunol. 2013;4:289.

9. Hetland ML. DANBIO--powerful research database and electronic patient record. Rheumatology (Oxford). 2011;50(1):69-77.

10. Bank $\mathrm{S}$, et al. High-quality and -quantity DNA extraction from frozen archival blood clots for genotyping of single-nucleotide polymorphisms. Genet Test Mol Biomarkers. 2013;17(6):501-3.

11. Andersen V, et al. Polymorphisms in NF-kappaB, PXR, LXR, PPARgamma and risk of inflammatory bowel disease. World J Gastroenterol. 2011;17(2):197-206.

12. Ernst A, et al. Common polymorphisms in the microsomal epoxide hydrolase and $\mathrm{N}$-acetyltransferase 2 genes in association with inflammatory 
bowel disease in the Danish population. Eur J Gastroenterol Hepatol. 2011; 23(3):269-74.

13. Andersen $\mathrm{V}$, et al. Cyclooxygenase-2 (COX-2) polymorphisms and risk of inflammatory bowel disease in a Scottish and Danish case-control study. Inflamm Bowel Dis. 2011;17(4):937-46.

14. Andersen $\mathrm{V}$, et al. The polymorphism rs3024505 proximal to IL-10 is associated with risk of ulcerative colitis and Crohns disease in a Danish case-control study. BMC Med Genet. 2010;11:82.

15. Ernst $\mathrm{A}$, et al. Genetic variants of glutathione S-transferases mu, theta, and pi display no susceptibility to inflammatory bowel disease in the Danish population. Scand J Gastroenterol. 2010;45(9):1068-75.

16. Ostergaard $\mathrm{M}$, et al. Cyclooxygenase-2, multidrug resistance 1, and breast cancer resistance protein gene polymorphisms and inflammatory bowel disease in the Danish population. Scand J Gastroenterol. 2009;44(1):65-73.

17. Ernst A, et al. Mutations in CARD15 and smoking confer susceptibility to Crohn's disease in the Danish population. Scand J Gastroenterol. 2007:42(12):1445-51.

18. Exarchou S, et al. The prevalence of clinically diagnosed ankylosing spondylitis and its clinical manifestations: a nationwide register study. Arthritis Res Ther. 2015;17:118.

19. Manolova I, et al. Association of single nucleotide polymorphism at position -308 of the tumor necrosis factor-alpha gene with ankylosing spondylitis and rheumatoid arthritis. Biotechnol Biotechnol Equip. 2014;28(6):1108-14.

20. Hohler T, et al. Association of different tumor necrosis factor alpha promoter allele frequencies with ankylosing spondylitis in HLA-B27 positive individuals. Arthritis Rheum. 1998;41(8):1489-92.

21. McGarry F, et al. The -308.1 polymorphism in the promoter region of the tumor necrosis factor gene is associated with ankylosing spondylitis independent of HLA-B27. J Rheumatol. 1999;26(5):1110-6.

22. Milicic A, et al. Interethnic studies of TNF polymorphisms confirm the likely presence of a second MHC susceptibility locus in ankylosing spondylitis. Genes Immun. 2000;1 (7):418-22.

23. Zhao $\mathrm{S}$, et al. The association of NLRP3 and TNFRSF1A polymorphisms with risk of ankylosing spondylitis and treatment efficacy of etanercept. J Clin Lab Anal. 2017;31(6). https://doi.org/10.1002/jcla.22138. Epub 23 Jan 2017.

24. Abdollahi E, et al. Protective role of R381Q (rs1 1209026) polymorphism in IL-23R gene in immune-mediated diseases: a comprehensive review. J Immunotoxicol. 2016;13(3):286-300.

25. Roberts AR, et al. Investigation of a possible extended risk haplotype in the IL23R region associated with ankylosing spondylitis. Genes Immun. 2017; 18(2):105-8.

26. Rueda B, et al. The IL23R Arg381GIn non-synonymous polymorphism confers susceptibility to ankylosing spondylitis. Ann Rheum Dis. 2008;67(10):1451-4.

27. Rahman $P$, et al. Association of interleukin-23 receptor variants with ankylosing spondylitis. Arthritis Rheum. 2008;58(4):1020-5.

28. Karaderi $\mathrm{T}$, et al. Association between the interleukin 23 receptor and ankylosing spondylitis is confirmed by a new UK case-control study and meta-analysis of published series. Rheumatology (Oxford). 2009; 48(4):386-9.

29. Safrany E, et al. Variants of the IL23R gene are associated with ankylosing spondylitis but not with Sjogren syndrome in Hungarian population samples. Scand J Immunol. 2009;70(1):68-74.

30. Duan Z, et al. Interleukin-23 receptor genetic polymorphisms and ankylosing spondylitis susceptibility: a meta-analysis. Rheumatol Int. 2012;32(5):1209-14.

31. Lee $\mathrm{YH}$, et al. Associations between interleukin-23R polymorphisms and ankylosing spondylitis susceptibility: a meta-analysis. Inflamm Res. 2012; 61(2):143-9.

32. Brionez TF, Reveille JD. The contribution of genes outside the major histocompatibility complex to susceptibility to ankylosing spondylitis. Curr Opin Rheumatol. 2008;20(4):384-91.

33. Burton PR, et al. Association scan of 14,500 nonsynonymous SNPs in four diseases identifies autoimmunity variants. Nat Genet. 2007;39(11):1329-37.

34. Wang W, et al. Association between protein tyrosine phosphatase nonreceptor type 22 (PTPN22) polymorphisms and risk of Ankylosing spondylitis: a meta-analysis. Med Sci Monit. 2017;23:2619-24.

35. Bank, S, et al. Polymorphisms in the inflammatory pathway genes TLR2, TLR4, TLR9, LY96, NFKBIA, NFKB1, TNFA, TNFRSF1A, IL6R, IL10, IL23R, PTPN22, and PPARG are associated with susceptibility of inflammatory bowel disease in a Danish cohort. PLoS One. 2014;9(6):e98815.

36. Bank, S, et al. Polymorphisms in the toll-like receptor and the IL-23/LL-17 pathways were associated with susceptibility to inflammatory bowel disease in a Danish cohort. PLoS One. 2015;10(12):e0145302.
37. Bank S, et al. Associations between functional polymorphisms in the NFkappaB signaling pathway and response to anti-TNF treatment in Danish patients with inflammatory bowel disease. Pharmacogenomics J. 2014;14(6):526-34.

38. Bank S, et al. Genetically determined high activity of IL-12 and IL-18 in ulcerative colitis and TLR5 in Crohns disease were associated with nonresponse to anti-TNF therapy. Pharmacogenomics J. 2018;18(1):87-97.

39. Bank $\mathrm{S}$, et al. Effectiveness of anti-tumour necrosis factor-alpha therapy in Danish patients with inflammatory bowel diseases. Dan Med J. 2015;62(3): A4994.

40. Bank S. A cohort of anti-TNF treated Danish patients with inflammatory bowel disease, used for identifying genetic markers associated with treatment response. Dan Med J. 2015;62(5):B5087.

41. Sode J, et al. Anti-TNF treatment response in rheumatoid arthritis patients is associated with genetic variation in the NLRP3-inflammasome. PLOS One. 2014;9(6):e100361.

42. Sode J, et al. Genetic variations in pattern recognition receptor loci are associated with anti-TNF response in patients with rheumatoid arthritis. PLoS One. 2015;10(10):e0139781.

43. Sode J, et al. Confirmation of an IRAK3 polymorphism as a genetic marker predicting response to anti-TNF treatment in rheumatoid arthritis. Pharmacogenomics J. 2018;18(1):81-6.

44. Loft ND, et al. Associations between functional polymorphisms and response to biological treatment in Danish patients with psoriasis. Pharmacogenomics J. 2018;18(3):494-500.

45. Bek S, et al. Systematic review: genetic biomarkers associated with anti-TNF treatment response in inflammatory bowel diseases. Aliment Pharmacol Ther. 2016;44(6):554-67.

46. Bek S, et al. Systematic review and meta-analysis: pharmacogenetics of antiTNF treatment response in rheumatoid arthritis. Pharmacogenomics J. 2017; 17(5):403-11.

47. Loft ND, et al. Genetic polymorphisms associated with psoriasis and development of psoriatic arthritis in patients with psoriasis. PLoS One. 2018; 13(2):e0192010.

48. Oliveira JM, et al. The -308 bp TNF gene polymorphism influences tumor necrosis factor expression in leprosy patients in Bahia state, Brazil. Infect Genet Evol. 2016;39:147-54.

49. Kaluza W, et al. Different transcriptional activity and in vitro TNF-alpha production in psoriasis patients carrying the TNF-alpha 238A promoter polymorphism. J Invest Dermatol. 2000;114(6):1180-3.

50. Wang $\mathrm{GB}$, et al. A regulatory polymorphism in promoter region of TNFR1 gene is associated with Kawasaki disease in Chinese individuals. Hum Immunol. 2011;72(5):451-7.

51. Kariuki SN, Crow MK, Niewold TB. The PTPN22 C1858T polymorphism is associated with skewing of cytokine profiles toward high interferon-alpha activity and low tumor necrosis factor alpha levels in patients with lupus. Arthritis Rheum. 2008;58(9):2818-23.

52. Oosting $\mathrm{M}$, et al. Role of interleukin-23 (IL-23) receptor signaling for IL-17 responses in human Lyme disease. Infect Immun. 2011;79(11):4681-7.

53. Jaiswal PK, et al. Association of IL-12, IL-18 variants and serum IL-18 with bladder cancer susceptibility in north Indian population. Gene. 2013;519(1):128-34.

54. Dziedziejko V, et al. The impact of IL18 gene polymorphisms on mRNA levels and interleukin-18 release by peripheral blood mononuclear cells. Postepy Hig Med Dosw (Online). 2012;66:409-14.

55. Sherlock JP, et al. IL-23 induces spondyloarthropathy by acting on ROR-gammat+ CD3+CD4-CD8- entheseal resident T cells. Nat Med. 2012;18(7):1069-76.

56. Uciechowski P, et al. Susceptibility to tuberculosis is associated with TLR1 polymorphisms resulting in a lack of TLR1 cell surface expression. J Leukoc Biol. 2011;90(2):377-88.

57. Gu W, et al. Functional significance of gene polymorphisms in the promoter of myeloid differentiation-2. Ann Surg. 2007;246(1):151-8.

58. Maxwell $L$, et al. TNF-alpha inhibitors for ankylosing spondylitis. Cochrane Database Syst Rev. 2015;18(4):Cd005468.

59. Pavelka K, et al. Efficacy, safety, and tolerability of secukinumab in patients with active ankylosing spondylitis: a randomized, double-blind phase 3 study, MEASURE 3. Arthritis Res Ther. 2017;19(1):285.

60. Cheung PP. Anti-IL17A in axial Spondyloarthritis-where are we at? Front Med (Lausanne). 2017:4:1.

61. Jandus $C$, et al. Increased numbers of circulating polyfunctional Th17 memory cells in patients with seronegative spondylarthritides. Arthritis Rheum. 2008;58(8):2307-17. 
62. Singh R, Aggarwal A, Misra R. Th1/Th17 cytokine profiles in patients with reactive arthritis/undifferentiated spondyloarthropathy. J Rheumatol. 2007; 34(11):2285-90.

63. Xueyi $L$, et al. Levels of circulating Th17 cells and regulatory $T$ cells in ankylosing spondylitis patients with an inadequate response to antiTNF-alpha therapy. J Clin Immunol. 2013;33(1):151-61.

64. Londono J, et al. The association between serum levels of potential biomarkers with the presence of factors related to the clinical activity and poor prognosis in spondyloarthritis. Rev Bras Reumatol. 2012;52(4):536-44.

65. Karimi $\mathrm{M}$, et al. A critical assessment of the factors affecting reporter gene assays for promoter SNP function: a reassessment of -308 TNF polymorphism function using a novel integrated reporter system. Eur J Hum Genet. 2009;17(11):1454-62.

66. Ibfelt EH, et al. Validity and completeness of rheumatoid arthritis diagnoses in the nationwide DANBIO clinical register and the Danish National Patient Registry. Clin Epidemiol. 2017;9:627-32.

67. Gast A, et al. Association of inherited variation in toll-like receptor genes with malignant melanoma susceptibility and survival. PLoS One. 2011;6(9): e24370.

68. Zhang F, et al. Polymorphisms in toll-like receptors 2, 4 and 5 are associated with legionella pneumophila infection. Infection. 2013;41(5):941-8.

69. Chen $\mathrm{H}$, et al. Single nucleotide polymorphisms in the human interleukin-1B gene affect transcription according to haplotype context. Hum Mol Genet. 2006;15(4):519-29.

70. Yoshida M, et al. Haplotypes in the expression quantitative trait locus of interleukin-1 beta gene are associated with schizophrenia. Schizophr Res. 2012;140(1-3):185-91.

71. Wen $\mathrm{AQ}$, et al. Clinical relevance of IL-1 beta promoter polymorphisms $(-1470,-511$, and -31$)$ in patients with major trauma. Shock. 2010;33(6): $576-82$.

72. Lind $\mathrm{H}$, Haugen A, Zienolddiny S. Differential binding of proteins to the IL1B -31 T/C polymorphism in lung epithelial cells. Cytokine. 2007;38(1):43-8.

Ready to submit your research? Choose BMC and benefit from:

- fast, convenient online submission

- thorough peer review by experienced researchers in your field

- rapid publication on acceptance

- support for research data, including large and complex data types

- gold Open Access which fosters wider collaboration and increased citations

- maximum visibility for your research: over $100 \mathrm{M}$ website views per year

At $\mathrm{BMC}$, research is always in progress.

Learn more biomedcentral.com/submissions 